\title{
Research on Evaluation Model of Situational Awareness for The Grid Information Network
}

\author{
ZHANG Huiwen, BAO Guangyu, ZHANG Yi, LV Zonghang \\ College of Command Information System, PLA Science and Technology University \\ NanJing, China \\ e-mail: feileha@163.com
}

\begin{abstract}
The components of situational awareness for the grid information network are analyzed, and models for evaluating the ability of situational awareness are established both in the information domain and the cognitive domain. In the information domain, three aspects of information quantity including completeness, accuracy and timeliness are proposed to evaluate the situational awareness ability. In the cognitive domain, the Logistic equation is established in combination with the quantitative analyses of the information domain in order to describe the change of the situational awareness from time to time.
\end{abstract}

Keywords-the grid information network; situational awareness; completeness; accuracy; timeliness; Logistic.

\section{INTRODUCTION}

Comprehensive, accurate and immediate battlefield situation awareness allows commanders to have a better understanding for the battlefield environment and situations. The military is going to establish the grid information network during the next five years. It is valuable to study the situational awareness for it reflects the ability of grid information network on gathering, processing and the distribution

This paper uses information quality and Logistic equation respectively to make quantitative model of situational awareness for each sensing unit both in the information domain and the cognitive domain, which provid general method for the evaluation of situational awareness.

\section{FORMATION OF THE SITUATIONAL AWARENESS FOR THE GRID INFORMATION NETWORK}

Grid network is able to gather, to process, to store, to distribute and to manage the information in accordance with the requirements of different people, and its main feature is "network-based information service". The grid information network provides the supporting environment for the development and operation of each level of military users, it can greatly meet the needs while in information retrieval, transmission, processing, storage, distribution, application, management and the security for all-level command personnel, fighter and the security personnel, so it is a key in the IT-based warfare to seize the information advantage, the decision advantage and the battlefield advantage. The main capabilities of the grid information network include: the integrated network communication capability, the grid computing and storage capacity, the networked information service and the insurance capability. The specific content is showed in Figure 1.

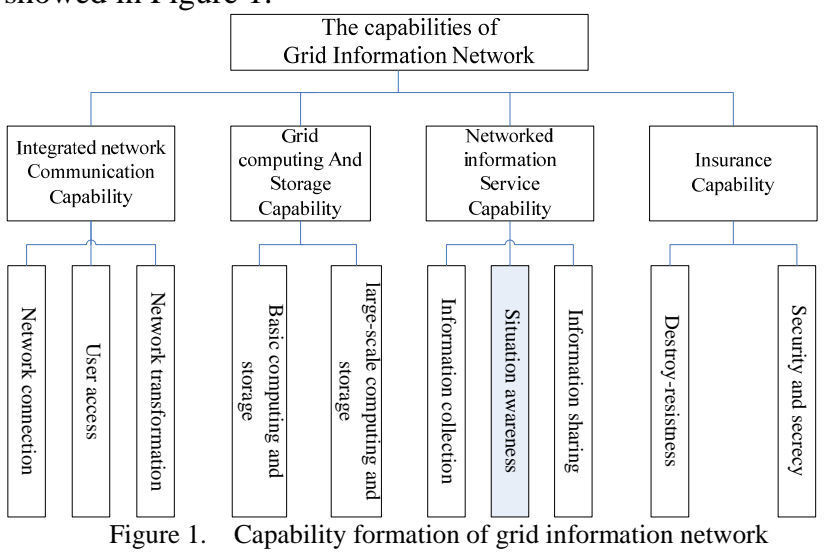

The situational awareness capability is one of parts to constitute the networked information service capability and it plays an important role in the grid information network. The situational awareness is to perceive all useful elements that human cares and to understand their intention, as well as the quick recognition of their identity [2]. The grid information network will start the situational awareness through the sensing unit, three steps of awareness, understanding and the prediction are needed in the process, see the capability formation shown in Figure 2.

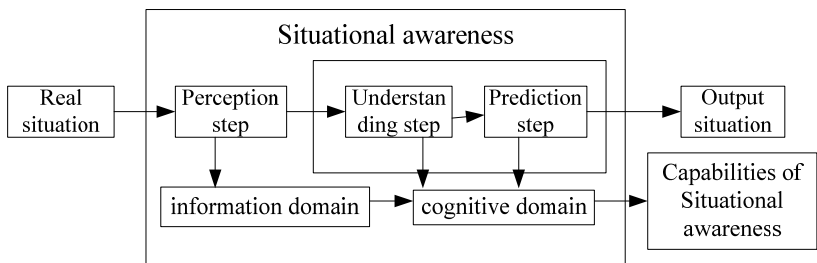

Figure 2. The formation of situational awareness process and the capability

The "real situation" in the figure is the real situation of target elements, and the "output situation" is the situation condition that the system outputted after a series of perception processes. Generally speaking, there are some differences between the "output situation" and the "real situation". A complete situational awareness needs to experience three steps of perception, understanding and the prediction. The perception step shows the transformation that the information from physical domain to the information domain, showing as a capability of information 
domain; The understanding and the prediction steps mean that the information transforms from the information domain to the cognitive domain, representing as a capability of cognitive domain. These steps are interlocking and forming the process of situational awareness together; however, the corresponding quality of situational awareness capability decides the consistency level between the real situation and the output situation. Therefore, how to quantitatively measure the situational awareness provides an important basis for the effect evaluation and the follow-up command decision-making.

\section{CAPABILITY EVALUATION OF SITUATIONAL AWARENESS}

The current evaluation situational awareness capability needs to achieve from the information domain and the cognitive domain. The information domain is used to measure the consistency between the situation information and the information of practical battlefield situation; the cognitive domain is mainly used to measure the consistency between commanders' perception and deduction over current situation and the real situation which belongs to a high-level perception. The paper is going to respectively evaluate the situational awareness from the information domain and the cognitive domain.

\subsection{Indicator Evaluation on Situational Awareness Capability of the Information Domain}

Generally speaking, both the real situation and the output situation can be described by three elements, namely the target number, the target feature and the target time [3]. Therefore, we can choose the completeness, the accuracy and the effectiveness of the information quality in accordance with the needs and the feature of the battle as the evaluation. We can establish the relevant indicator to measure the consistency of three elements between real situation and the output situation and build the evaluation model of situational awareness capability.

\section{(1) Completeness}

Completeness is the coincidence degree that between the practical target number of the real situation and the target number of the perception situation.

The target is usually divided into several levels according to the importance. We suppose there are $\mathrm{N}$ different level of targets in specified target zone, and the perception information shows the enemy targets of the zone, there are $E_{1}$ of level-1 targets, $E_{2}$ of level-2 targets... $E_{N}$ of level-N; however the battlefield situational awareness correctly perceived that there are $E_{1}^{\prime}$ of level-1 targets, $E_{2}^{\prime}$ of level-2 targets... $E_{N}^{\prime}$ of level-N targets. As a result, the completeness $\beta$ in the situational awareness can be defined as follow:

$$
\left\{\begin{array}{l}
\beta=\sum_{i=1}^{N}\left(a_{i} \frac{E_{i}^{\prime}}{E_{i}}\right) \\
\sum_{i=1}^{N} a_{i}=1
\end{array}\right.
$$

In the equation, $a_{1}, a_{2} \ldots$ and $a_{N}$ are successively the weightiness that the target of level-1,level-2... and the level-N perform in the situational awareness.

\section{(2) Accuracy}

The accuracy refers to the coincidence degree between the feature of enemy targets and the real targets in the specified task zone.

Use the eigenvector of the target information to indicate the status of a certain task. We suppose $G_{j}$ and $P_{j}$ respectively represent the eigenvector of $j$ targets in the real situation and the output situation, then $G_{j}$ and $P_{j}$ can be respectively represented as follow:

$$
\begin{array}{cl}
G_{j}=\left[g_{j k}\right] & k \in\{1,2, \cdots, n\} \\
P_{j}=\left[p_{j k}\right] & k \in\{1,2, \cdots, n\}
\end{array}
$$

The elements of the eigenvector are the poison and speed of jth target, $\mathrm{k}$ is the jth target's kth characteristic parameter, and $n$ is the number of jth target's characteristic parameter. So, the deviation definition between the eigenvector of real situation and the eigenvector of output situation can be defined as follow:

$$
R_{j k}=\left|p_{j k}-g_{j k}\right|
$$

Therefore, the average deviation degree $\gamma_{j}$ between the eigenvector of jth target in the output situation and the real situation can be defined as follow:

$$
\gamma_{j}=\sum_{k=1}^{n} \frac{R_{j k}}{g_{j k}} / n
$$

We suppose that the output situation in a certain task has correctly found $\mathrm{M}$ enemy belonging to $\mathrm{N}$ level. So the average accuracy $\gamma$ of output situation can be defined as:

$$
\left\{\begin{array}{l}
\gamma=\sum_{i=1}^{N}\left[b_{i} \sum_{j \in J_{i}}\left(\frac{1-\gamma_{j}}{\left|J_{i}\right|}\right)\right] \\
\sum_{i=1}^{N}\left|J_{i}\right|=M \\
\sum_{i=1}^{N} b_{i}=1
\end{array}\right.
$$

The $b_{i}$ is the weightiness that level-i target perform in the accuracy of battlefield situational awareness; $J_{i}$ 
represents the ith target, and $\left|J_{i}\right|$ represents the number of level-I target.

\section{(3) Timeliness}

The timeliness describes the degree that information retrieval meets the time requirements of the task. Since the opportunity is fleeting in the battlefield, so the information receives more quickly, then it can be timely processed. Here we are not considering the problem of information overload.

Obviously, if there is no delay in the process of information retrieval (namely the delay is 0 ), then the timeliness is the best and its value equals 1 ; with the increase of delay, accordingly the timeliness will be gradually reduced; If the delay has exceeded the required task time, than we will lose the fight opportunity and the timeliness will be 0 . As a result, we set a $\mathrm{T}$ as the critical time, and all information will be meaningless if they exceed this $\mathrm{T}$. We suppose the delay to finish a task is $\Delta t$, and suppose $\eta$ to represents timeliness, then it can be defined as follow:

$$
\eta=1-\Delta t / T
$$

In order to guarantee the timeliness, we define it as $0 \leq \Delta t \leq T$.

\subsection{The Evaluation Model for the Situational Awareness of Cognitive Domain}

In the cognitive process, the sensing unit will understand through the pre-collected information in order to get to understand the battlefield situation, enemy's intention and the confrontation situation [2]. The past studies on the situational awareness of cognitive domain has defined the key elements, and the characters and the tasks were described by formalized language [4], and some situational awareness processes were regarded as a process of knowledge generated by the information, and the knowledge generation mechanism was described by the method of information theory [1]. But these methods have not made a effective fusion with the situational awareness process of information domain.

Due to the cognitive evolution process has the feature of Logistic process [5-7], in this section we use the Logistic equation to establish situational awareness model lf single sensing unit to describe the situational awareness capability by quantifying the quality of situational awareness of cognitive domain. The main factors that affect the quality of situational awareness of cognitive domain depend on the quality of sensing unit and the quality of front-end information acquisition (including completeness, accuracy and the timeliness). Therefore, we can regard the quality of situational awareness of cognitive domain as a function of $t$ to combine with the three indicators that evaluated the information domain and establish the situational awareness model of the sensing unit.

The element to describe the situational awareness of cognitive domain is the cognitive capability of sensing unit, it directly decides the change of cognitive domain capability, and here we suppose the cognitive capability of sensing unit is invariable. Since the timeliness $\eta$ is only working on the information domain, and we consider that the timeliness meets the requirements while describing the situational awareness capability of cognitive domain. So here we suppose the information domain has acquired enough information within the requirements of timeliness in a certain task, in other words, there are determined values for the information accuracy and the information completeness of sensing unit. Then we use the cognitive capability, the completeness and the accuracy of information as parameters to establish a Logistic equation, and here we got the quality of situational awareness at $\mathrm{t}$ moment as follow:

$$
S(t)=\frac{\alpha \beta \gamma}{1+e^{-\alpha \beta \gamma(t-d)}}
$$

The meaning of each parameter as follow:

$\alpha$ - Cognitive capability of sensing unit. $\alpha$ can use the sensing unit to get the correct probability of situational awareness, because there must be limits in human's cognition to the objects, so the situational awareness cannot be understood $100 \%$, so here we got $\alpha \in[0,1)$.

$\beta$ - The information completeness that the sensing unit independently acquired, namely the portion that useful part in the total required information of the task. $\beta \in[0,1]$ means the independently acquired information of sensing unit might be incomplete to finish the task.

$\gamma \_$The information accuracy that independently acquired by the sensing unit. There must be some technical limits in information acquisition, so here we got $\gamma \in[0,1)$

$d$ - The translation distance of the curve to confirm the value while the curve reach at $\mathrm{t}=0$.

In the equation, $t$ is an independent variable, the value of $S(t)$ will accordingly increase as $t$ increases. Under the situation of a certain battlefield or task, there is a time limit for the process of situational awareness. The situational awareness capability of cognitive domain of information basic network can be reflected as the maximum of $S(t)$, the $S(t)$ is bigger, the capability is stronger, the value of $S(t)$ is related to the each parameters of the equation. According to the equation (4), we know $\alpha \beta \gamma$ together decide the maximum of situational awareness of sensing unit, additionally they also decide the speed to reach the maximum of situational awareness.

Combine the analysis of section 3.1 and 3.2, we show the evaluation process of situational awareness of the information domain and the cognitive domain, see as figure 3. 


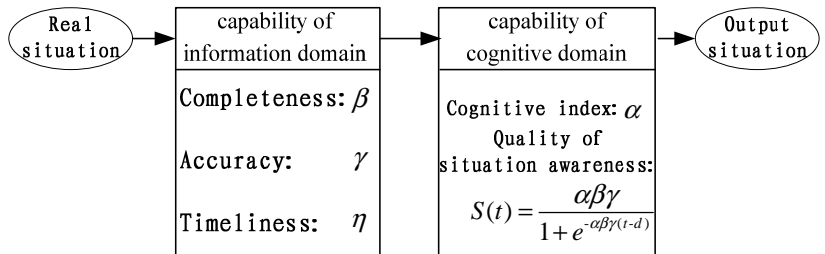

Figure 3. The evaluation process for the situational awareness of information domain and the cognitive domain

From the figure we know that the completeness $\beta$, accuracy $\gamma$ and the timeliness $\eta$ of the system was measured; and we can get the evaluation results for the situational awareness of cognitive domain by substituting $\alpha, \beta$ and $\gamma$ into the equation.

\section{SIMULATION AND ANALYSIS}

To verify the effectiveness of the above evaluation model, here we make a simulation for the change of situational awareness quality as follow.

We suppose there are two level of targets, 5 level-1 targets and 4 level-2 targets, namely $E_{1}=5$ and $E_{2}=4$; however the sensing unit correctly perceived that there are 3 level-1 targets and 3 level-2 targets, namely $E_{1}^{\prime}=3$ and $E_{2}^{\prime}=3$. The weightiness of level-1 target and the level-2 target are $a_{1}=0.6$ and $a_{2}=0.4$; the weightiness of sensing unit are $b_{1}=0.7$ and $b_{2}=0.3$.Define the critical time to achieve the task as $T=60 \mathrm{~s}$, the critical time of sensing unit is $\Delta t=30 \mathrm{~s}$. Define the cognitive capability of sensing unit as $\alpha=0.8$.

According to the completeness evaluation model described in equation (1), the completeness will be:

$$
\beta=a_{1} \frac{E_{1}^{\prime}}{E_{1}}+a_{2} \frac{E_{2}^{\prime}}{E_{2}}=0.6 \cdot \frac{3}{5}+0.4 \cdot \frac{3}{4}=0.66
$$

We suppose the perceived normalization eigenvectors of real situation and the output situation of these 6 targets as follow in Table 1:

TABLE 1. EACH NORMALIZATION EIGENVECTOR OF REAL SITUATION AND THE OUTPUT SITUATION.

\begin{tabular}{|c||c|c|c|}
\hline & Numb. & Real situation $G_{j}$ & Output situation $P_{j}$ \\
\hline \hline \multirow{2}{*}{$\begin{array}{c}\text { Level-1 } \\
\text { target }\end{array}$} & 1 & $(1.5,2.0,2.9)$ & $(1.4,2.1,3.1)$ \\
\cline { 2 - 4 } & 2 & $(2.1,0.5,1.3)$ & $(1.9,1.0,1.5)$ \\
\cline { 2 - 4 } & 3 & $(1.6,4.5,2.5)$ & $(2.0,4.2,2.7)$ \\
\hline \multirow{2}{*}{$\begin{array}{c}\text { Level-2 } \\
\text { target }\end{array}$} & 4 & $(1.0,1.5)$ & $(1.4,2.1)$ \\
\cline { 2 - 4 } & 5 & $(2.0,2.5)$ & $(1.9,2.0)$ \\
\cline { 2 - 4 } & 6 & $(1.4,1.8)$ & $(2.0,2.7)$ \\
\hline
\end{tabular}

According to the perceived weightiness of these two level targets in the situation and the accuracy definition described in equation (2), then the accuracy can be represented as follow:

$$
\begin{aligned}
\gamma= & b_{1}\left[\frac{\left(1-\gamma_{1}\right)+\left(1-\gamma_{2}\right)+\left(1-\gamma_{3}\right)}{3}\right] \\
& +b_{2}\left[\frac{\left(1-\gamma_{4}\right)+\left(1-\gamma_{5}\right)+\left(1-\gamma_{6}\right)}{3}\right] \\
= & 0.61
\end{aligned}
$$

And according to the equation (3), the timeliness will be:

$$
\eta=1-\Delta t / T=1-30 / 60=0.5
$$

Due to $\alpha=0.8$, combining calculated value of the above $\beta$ and $\gamma$, according to logistic equation to draw up the trend chart of quality of situational awareness along with the time, see as Figure 4.

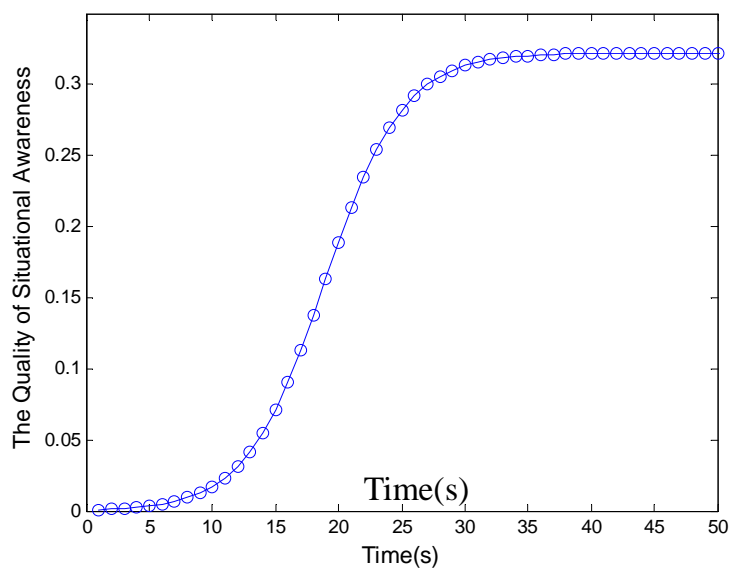

Figure 4 . The trend graph of the quality of situational awareness varying with the time.

We can see with the increase of $t$, the quality of situational awareness is accordingly increasing and the speed perform the trend of FAST---SLOW---FAST which correspond with human's cognition on the objects.

Here we choose different values for $\beta$ and $\gamma$, and calculate the cognitive capability of situational awareness under different condition of information domain and the cognitive domain, and we analyzed the effects that different values would make on the situational awareness capability of cognitive. Define $\alpha=0.8$ as invariable, find different values of $\beta$ when $\gamma=0.61$, and accordingly observe $\mathrm{S}(\mathrm{t})$ value while $\beta=0.66$ and the different value of $\gamma$. See the simulation result as Figure 5 . 

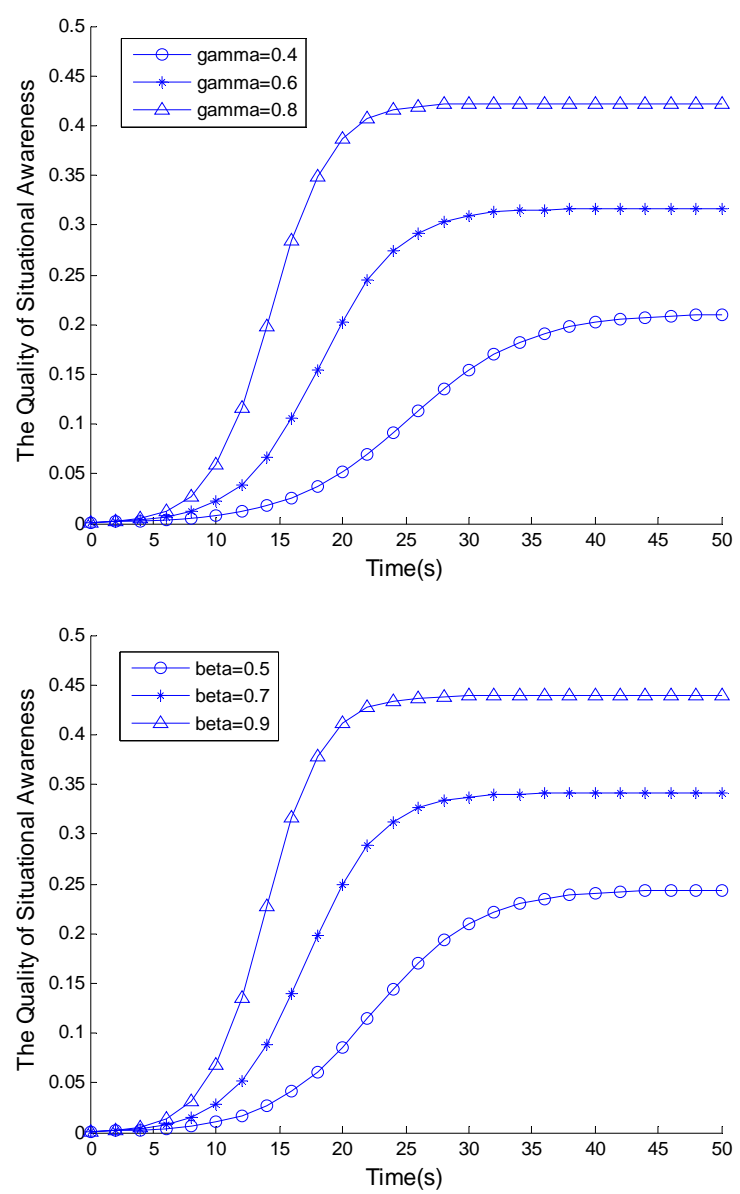

Figure 5. The verification trend of situational awareness when parameters are found in different values

We can see that the accuracy $\beta$ and the completeness $\gamma$ would both decide the increasing speed of the quality of situational awareness. If the value of completeness $\beta$ is bigger, the final quality of situational awareness is bigger. In a similar way, the accuracy $\gamma$ is also suitable for this conclusion.

\section{CONCLUSION}

According to the feature of information warfare, this paper makes models of situational awareness capability for the grid information network both in the information domain and the cognitive domain. In the information domain, we make evaluation for situational awareness capability through the target number, target feature and the target time; in the cognitive domain, we establish the Logistic situational awareness equation to describe the effects of different parameters on the quality of situational awareness. The simulation results shows the information accuracy and the information completeness are in proportion to the maximum and the speed of the quality of situational awareness. This method is a new way of thinking for the evaluation of situational awareness capability of grid information network.

\section{REFERENCES}

[1] Moffat J. Complexity Theory and network centric warfare[M]. Washington DC, USA: CCRP Publication Series, 2003.

[2] SHI Yuedong, Command and decision of strategy. BeiJing: People's Liberation Army Press, 2005

[3] NIE Zhong, Huang Gaoming. Quantitative Analysis on Sea Battlefield Cyberspace Situation Awareness Capability[J].Shipboard Electronic Countermeasure. 2011, 34(6): 39-41.

[4] LIU Xiaoping, Shi hui. Cooperative Awareness model based on information visualization[J].Joumal on Communications. 2006, 27(11): 24-30.

[5] CHEN Lixin. Effectiveness Evaluation of Network-Centric Air-Defense Missile Systems. BeiJing: National Defense Industry Press, 2007.

[6] ZHANG Ruilong, Li Mingyong. Situation Awareness to Decision Maker in Coalition Group[J]. Ship Electronic Engineering, 2008, 28(5): 70-74.

[7] FEI Aiguo, Wang Xinhui. Measurement and Effectiveness Evaluation of Information superiority. BeiJing: Military Science Press, 2006. 